\title{
Current and planned research on agriculture for improved nutrition: A mapping and a gap analysis
}

\author{
J. Waage ${ }^{1}$, C. Hawkes ${ }^{1}$, R. Turner ${ }^{1}$, E. Ferguson ${ }^{1}$, D. Johnston ${ }^{1}$, B. Shankar ${ }^{1}$, G. McNeill ${ }^{2}$, \\ J. Hussein ${ }^{2}$, H. Homans ${ }^{3}$, D. Marais ${ }^{2}$ and F. Haseen ${ }^{2}$ \\ ${ }^{1}$ Leverhulme Centre for Integrative Research on Agriculture and Health (LCIRAH), London, UK, ${ }^{2}$ Division of Applied \\ Health Sciences, University of Aberdeen, UK and ${ }^{3}$ Centre for Sustainable International Development, University of \\ Aberdeen, UK
}

Despite the clear potential for agricultural change to improve nutrition in low and middle income countries (LMIC), the evidence base for this relationship is poor. Recent systematic reviews ${ }^{(1,2)}$ of studies which have evaluated agricultural interventions for improved nutrition reveal little strong evidence of impact and a need for more and better designed research. The study aimed to assist the development of a more coherent framework for research in this area to ultimately improve the overall quality of research on agriculture for improved nutrition.

A conceptual framework linking agriculture, food, and nutrition was developed by an expert panel making use of existing concepts for interactions between agriculture and health. It was used to define the domain of research on agriculture for improved nutrition, to establish inclusion criteria for research to be considered, and to characterise that research in a comparative way, relative to the different impact pathways. Mapping of current and planned research projects using snowballing and following specified inclusion and exclusion criteria was conducted from information obtained from the informants themselves and/or from project websites. A gap analysis was conducted to identify more poorly researched areas.

The study characterized 151 research projects, most of which are part of broader research programmes. A significant majority concern Sub-Saharan Africa, with a particular focus on nutritional impacts on women and children. Current research is of a range of types, with the majority $(58 \% ; n=88)$ involving research on some form of active intervention into agriculture and the rest $(n=63)$ involving research on existing datasets and 3 systematic reviews. The gap analysis on 100 of the projects with sufficient information identified eight clear research gaps: Full pathway of change especially not value chain; Indirect effect of changes in agriculture on nutrition; Effects of agricultural policy on nutrition; Governance, policy processes and political economy for development of agriculture-for-nutrition policies and programmes; Development of methodologies and appropriate metrics; Consumers as a broader target group especially rural workers and non-rural populations; Rural and urban poor at risk from nutrition-related noncommunicable diseases and Cost-effectiveness.

Most projects did not consider the value chain and few measure nutritional status. Very few projects considered indirect effects acting through economic outcomes. There is limited research underway on the development of new methodologies and metrics to measure effects along the different impact pathways, or to evaluate cost effectiveness of interventions.

This research was funded by DFID.

1. Masset E, Lawrence Haddad L, Cornelius A, Isaza-Castro J (2012) Effectiveness of agricultural interventions that aim to improve nutritional status of children: systematic review. $B M J 344, \mathrm{~d} 8222$.

2. Girard A, Self J, McAuliffe C, Oludea O (2012) The Effects of Household Food Production Strategies on the Health and Nutrition Outcomes of Women and Young Children: A Systematic Review. Paediatric and Perinatal Epidemiology 26(1), 205-222. 